\title{
State Estimation for Linear Discrete-Time Systems with Unknown Input Using Nonparametric Technique
}

\author{
V. Smagin \\ Departament of Applied Mathematics and Cybernetics \\ Tomsk State University \\ V. Udod \\ Departament of Economics \\ Tomsk State University
}

\begin{abstract}
The paper addressed the filtering problems with using nonparametric algorithms for discrete linear systems with the known control input, unknown input and parameters. The designed algorithms are based on combining the Kalman filter and nonparametric estimator. Examples are given to illustrate the usefulness of the proposed approach.
\end{abstract}

Keywords-kalman filter; discrete linear systems; unknown input; unknown parameters; filtering algorithm; nonparametric estimators

\section{INTRODUCTION}

An important issue of the Kalman filtering is the construction of algorithms for the class of discrete systems with unknown additive perturbations (input) and unknown parameters. Such systems are used as the models of real physical systems, as the models of objects with unknown errors, and for fault diagnostics and robust control.

The methods to estimate a state vector are often based on the LSM-estimator of unknown input [1-4]. In [5], such problem is solved with making use of compensation methods for linear stochastic systems with an unknown constant input. In [6], the problem is solved on fault diagnostic and faulttolerant control of systems with unknown input.

In this paper, for discrete linear stochastic systems with the known control input, an unknown input and parameters the algorith $m$ with use of the Kalman filtering and nonparametric estimators is proposed. This aproach generalizes the result of the paper [7]. The examples are given to illustrate the properties of the proposed algorithms in comparison with the algorith ms used the LSM-estimator.

\section{PROBLEM FORMULATION}

Consider the model of the linear discrete-time system with an unknown input and unknown parameters:

$$
\begin{gathered}
x(k+1)=(A+\Delta A(k)) x(k)+(B+\Delta B(k)) u(k)+f(k)+q(k), x(0)=x_{0}, \\
y(k)=H x(k)+v(k),
\end{gathered}
$$

where $x(k)$ is a state of the object, $u(k)$ is a known control input, $y(k)$ is an observation vector, $A$ and $H$ are the known matrices of the appropriate dimensions, $f(k)$ is an unknown

\author{
G. Koshkin \\ Departament of Applied Mathematics and Cybernetics \\ Laboratory of Geological Informatics of Computer Science \\ Department \\ Tomsk State University
}

input, $\Delta A(k)$ and $\Delta B(k)$ are matrices with the corresponding unknown elements (parameters). It is assumed that random perturbations $q(k)$ and noise measurements $v(k)$ are not correlated between themselves and are subject to the Gaussian distributions with the zero-vector means and the covariance matrices

$$
\mathrm{E}\left[q(k) q(t)^{\mathrm{T}}\right]=Q \delta(k, t), \quad \mathrm{E}\left[v(k) v(t)^{\mathrm{T}}\right]=V \delta(k, t),
$$

where $Q \geq 0$ and $V>0$ are the known matrices, $\delta(k, t)$ is the Kronecker symbol, E[·] denotes the expectation of a random variable. It is assumed that the vector of initial conditions $x_{0}$ is uncorrelated with values $q(k)$ and $v(k)$. The vector $x_{0}$ has the following characteristics:

$$
\mathrm{E}[x(0)]=\bar{x}_{0}, \mathrm{E}\left[\left(x(0)-\bar{x}_{0}\right)\left(x(0)-\bar{x}_{0}\right)^{\mathrm{T}}\right]=P_{0} .
$$

Here, we consider the case when prior knowledge about the time evolution of $f(k), \Delta A(k)$, and $\Delta B(k)$ are not available.

\section{FILTERING ALGORITHM}

As a filter estimating a state of object (1), we take the following filter:

$$
\begin{gathered}
\hat{x}(k+1)=A \hat{x}(k)+B u(k)+\hat{r}(k)+K(k)[y(k+1)- \\
H(A \hat{x}(k)+B u(k)+\hat{r}(k))], \hat{x}(0)=\bar{x}_{0}, \\
K(k)=P(k+1 / k) H^{\mathrm{T}}\left[H P(k) H^{\mathrm{T}}+V\right]^{-1}, \\
P(k+1 / k)=A P(k) A^{\mathrm{T}}+Q, \\
P(k+1)=(I-K(k) H) P(k+1 / k), P(0)=P_{0},
\end{gathered}
$$

where $\hat{x}(k)$ is a state estimator, $P(k)=\mathrm{E}\left[(x(k)-\hat{x}(k))(x(k)-\hat{x}(k))^{\mathrm{T}}\right], \hat{r}(k)$ is an estimator of the unknown vector

$$
r(k)=\Delta A(k) x(k)+\Delta B(k) u(k)+f(k) .
$$

However, formu las (3)-(6) can not be applied immed iately because $\hat{r}(k)$ is unknown. The estimator $\hat{r}(k)$ was obtained fro $m$ the criteria 


$$
J(r(k-1))=\mathrm{E}\left[\sum_{i=1}^{k}\|y(i)-H \hat{x}(i)\|_{C}^{2}+\|r(i-1)\|_{D}^{2}\right]
$$

Where \|\|$_{C}$ is the Euclidian norm, $C>0$ and $D>0$ are weight matrices.

Applying the mathematical induction, as in [7], we obtain the estimator of the unknown input

$$
\hat{r}(k)=\left(H^{\mathrm{T}} C H+D\right)^{-1} H^{\mathrm{T}} C \mathrm{E}[w(k)],
$$

Where $w(k)=y(k)-H A \hat{x}(k-1)$.

Now, let us estimate value $\mathrm{E}[w(k)]$ by nonparametric algorith ms [8]. Using the well known kernel estimates, we get

$$
\hat{r}(k)=S \hat{w}(k),
$$

where the $j$-th component of the vector takes the form

$$
\hat{w}_{j}(k)=\frac{\sum_{i=1}^{k} w_{j}(i) K_{j}\left(\frac{k-i+1}{h_{i, j}}\right)}{\sum_{i=1}^{k} K_{j}\left(\frac{k-i+1}{h_{i, j}}\right)} .
$$

In formula (9), $K_{j}(\cdot)$ is a kernel function, $h_{i, j}$ is a bandwidth parameter. We use the Gaussian kernels, and the bandwidths are calculated by the cross-validation method [9]

Also, we studied the properties of the filtering with another estimator $\hat{r}(k)=S \tilde{w}(k)$ for an unknown input (cf. [10]):

$$
\tilde{w}_{j}(k)=\sum_{i=1}^{k} w_{j}(i) K_{j}\left(\frac{k-i+1}{h_{i, j}}\right) \frac{\left(x_{i}-x_{i-1}\right)}{h_{i, j}} .
$$

\section{Simulation RESULTS}

In this section, we present simulation results to illustrate the performance of the proposed algorithm. Apply the filtering algorith m (3)-(6), using nonparametric estimates (9) and (10), to the model (1) and to the observations (2) with the following parameters:

$$
\begin{gathered}
A=\left(\begin{array}{cc}
0 & 1 \\
0.05 & 0.9
\end{array}\right), B=\left(\begin{array}{l}
1.0 \\
1.0
\end{array}\right), Q=\left(\begin{array}{cc}
0.01 & 0 \\
0 & 0.02
\end{array}\right), \\
V=\left(\begin{array}{cc}
0.8 & 0 \\
0 & 1.2
\end{array}\right), H=\left(\begin{array}{cc}
1.0 & 0 \\
0 & 1.0
\end{array}\right), P_{0}=\left(\begin{array}{cc}
1.0 & 0 \\
0 & 1.0
\end{array}\right), \\
C=\left(\begin{array}{cc}
1.0 & 0 \\
0 & 1.0
\end{array}\right), D=\left(\begin{array}{ll}
0 & 0 \\
0 & 0
\end{array}\right), \bar{x}_{0}=\left(\begin{array}{c}
3.5 \\
1
\end{array}\right) .
\end{gathered}
$$

Simulations were carried out with such input $f(k), u(k)$ and matrices $\Delta A(k), \Delta B(k)$ :

$$
f=\left(\begin{array}{l}
5 \\
2
\end{array}\right), u(k)=0.1, \Delta A=\left(\begin{array}{cc}
0 & 0.01 \\
-0.03 & 0.055
\end{array}\right), \Delta B(k)=\left(\begin{array}{l}
0.4 \\
0.7
\end{array}\right) .
$$

The proposed algorithms we comprise with the algorithms using the LSM-estimates from [2] by simulations. These comparisons are given in Figs. 1-4:
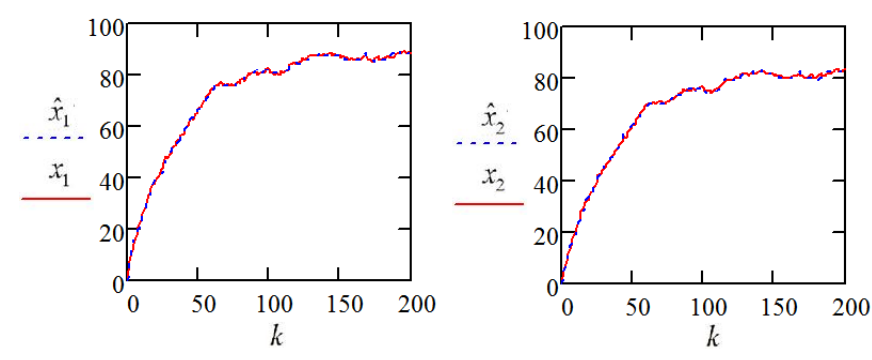

FIGURE I. THE DEPENDENCE OFTHE COMPONENTS ${ }^{x_{1}}, x_{2}$ AND THEIR EST IMATES $\hat{x}_{1}, \hat{x}_{2}$ WIT H NONPARAMET RIC ESTIMATES OF UNKNOWN INPUT. PLEASE, INSERT "OF” IN RED
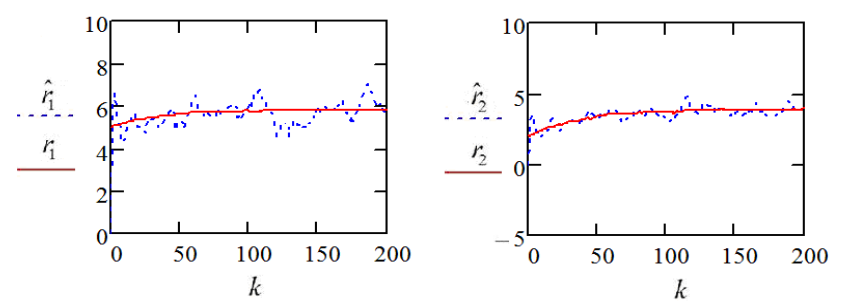

FIGURE II. THE EST IMATION OF UNKNOWN INPUT S WITH NONPARAMETRIC ESTIMATES (9).
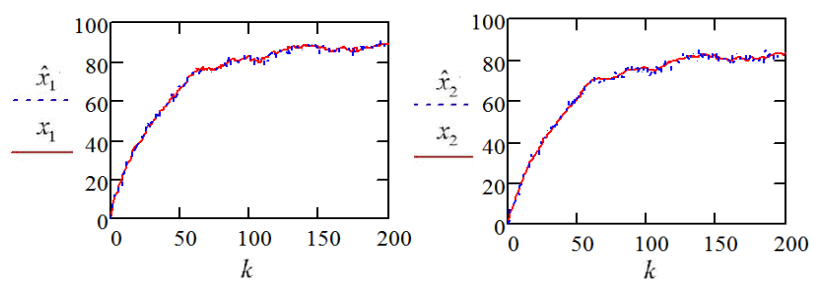

FIGURE III. THE DEPENDENCE OFTHE COMPONENTS $x_{1}, x_{2}$ AND THEIR EST IMATES $\hat{x}_{1}, \hat{x}_{2}$ WITH THE LSM-ESTIMATES.
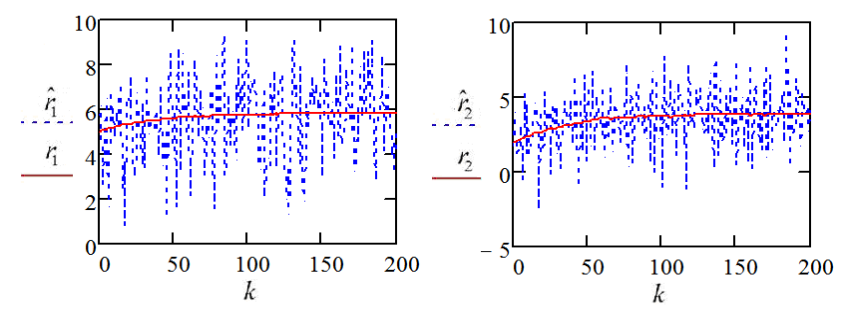

FIGURE IV. THE EST IMATION OF UNKNOWN INPUT S WITH THE LSM-EST IMATES.

We used the bandwidth parameters $h_{i, 1}=6, h_{i, 2}=8$ for all $i$. Below, in Tables 1 and 2 the standard errors

$\sigma_{x, i}=\sqrt{\frac{\sum_{k=1}^{N}\left(x_{i}(k)-\hat{x}_{i}(k)\right)^{2}}{N-1}}, \sigma_{r, i}=\sqrt{\frac{\sum_{k=1}^{N}\left(r_{i}(k)-\hat{r}_{i}(k)\right)^{2}}{N-1}}$
$(i=\overline{1,2})$ 
are presented for two filtering algorithms $(N=200)$ and by averaging 50 realizations.

TABLE I. STANDARD ERRORS FOR FLLTERING ALGORITHMS WITH NONPARAMETRICESTIMATES (9).

\begin{tabular}{|c|c|c|c|}
\hline$\sigma_{x, 1}$ & $\sigma_{x, 2}$ & $\sigma_{r, 1}$ & $\sigma_{r, 2}$ \\
\hline 0.963 & 0.992 & 0.524 & 0.345 \\
\hline
\end{tabular}

TABLE II. STANDARD ERRORS FOR FILTERING ALGORITHMS WITH THE LSM-ESTIMATES.

\begin{tabular}{|c|c|c|c|}
\hline$\sigma_{x, 1}$ & $\sigma_{x, 2}$ & $\sigma_{r, 1}$ & $\sigma_{r, 2}$ \\
\hline 1.471 & 1.561 & 1.988 & 2.263 \\
\hline
\end{tabular}

Figures and Tables show that the procedures with nonparametric estimates have the advantages in the accuracy in comparison with the known algorithms using the LSMestimates. It is seen that the presented nonparametric technique may be used in solving the general filtering problems with the known control input, unknown input and parameters. Applying the estimator (10) instead of (9) gives the similar simulation results in accuracy. The proposed algorithms can be used to synthesize the fault-tolerant and robust control.

\section{ACKNOWLEDGEMENTS}

Supported by Russian Foundation for Basic Research, projects 13-08-00744, 13-08-01015A, 13-08-98027, and Tomsk State University Competitiveness Improvement Program.

\section{REFERENCES}

[1] Hou, M. \& Patton, R. Optimal filtering for systems with unknown inputs, IEEE Trans. on Automat. Contr., 43, 1998, pp. 445-449.

[2] Janczak, D. \& Grishin, Y. State estimation of linear dynamic system with unknown input and uncertain observation using dynamic programming, Control and Cybemetics, 35(4), pp. 851-862, 2006.

[3] Gillijns, S. \& Moor, B. Unbiased minimum-variance input and state estimation for linear discrete-time systems, Automatica, 43, pp. 111-116, 2007.

[4] Hsien, C.-S. On the optimal of two-stage Kalman filter for systems with unknown input, Asian Journal of Control, 12(4), 2010, pp. 510-523.

[5] Smagin, V.I. State estimation for linear discrete systems with unknown input using compensations. Russian Physics Journal, 57(5), pp. 682-690, 2014.

[6] Witczak, M. Fault diagnosis and fault-tolerant control strategies for nonsystems. Chapter 2. Unknown input observers and filters, Lecture Notes in Electrical Engineering, Springer International Publishing, Switzerland, 2014, pp. 19-56.

[7] Koshkin, G.M. \& Smagin, V.I. Filtering and prediction for discrete systems with unknown input using nonparametric algorithms. Proc. $10^{\text {th }}$ Intemational Conference on Digital Technologies. Žilina, Slovakia. July 9-11, 2014, pp. 120-124.

[8] Dobrovidov, A., Koshkin, G. \& Vasiliev, V. Non-parametric state space models, Heber, UT 84032, USA. Kendrick Press, Inc. 2012.

[9] Leung, D. Cross-validation in nonparametric regression with outliers, Annals of Statistics, 33, 2005, pp. 2291-2310.
[10] Georgiev, A. Nonparametric kernel algorithm for recovery of function from noisy measurements with applications. IEEE Trans. Automatic Contr., 30(8), 1985, pp.782-784. 